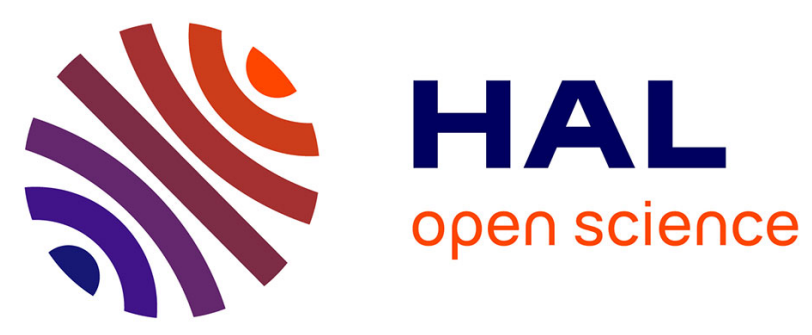

\title{
LA DIFFUSION D'UNE DÉMARCHE RSE INNOVANTE AU SEIN D'UN RÉSEAU DE PME: LE CAS DU CENTRE DES JEUNES DIRIGEANTS
}

Elise Bonneveux, Isabelle Calmé, Richard Soparnot

\section{To cite this version:}

Elise Bonneveux, Isabelle Calmé, Richard Soparnot. LA DIFFUSION D'UNE DÉMARCHE RSE INNOVANTE AU SEIN D'UN RÉSEAU DE PME: LE CAS DU CENTRE DES JEUNES DIRIGEANTS. Gestion 2000, 2011, 10.3917/g2000.282.0069 . hal-02429484

HAL Id: hal-02429484

https://hal.science/hal-02429484

Submitted on 6 Jan 2020

HAL is a multi-disciplinary open access archive for the deposit and dissemination of scientific research documents, whether they are published or not. The documents may come from teaching and research institutions in France or abroad, or from public or private research centers.
L'archive ouverte pluridisciplinaire HAL, est destinée au dépôt et à la diffusion de documents scientifiques de niveau recherche, publiés ou non, émanant des établissements d'enseignement et de recherche français ou étrangers, des laboratoires publics ou privés. 


\title{
LA DIFFUSION D'UNE DÉMARCHE RSE INNOVANTE AU SEIN D'UN RÉSEAU DE PME : LE CAS DU CENTRE DES JEUNES DIRIGEANTS
}

\author{
Elise Bonneveux, Isabelle Calme et Richard Soparnot
}

Association de recherches et publications en management | « Gestion 2000 »

2011/2 Volume 28 | pages 69 à 90

ISSN 0773-0543

Article disponible en ligne à l'adresse :

https://www.cairn.info/revue-gestion-2000-2011-2-page-69.htm

Distribution électronique Cairn.info pour Association de recherches et publications en management.

(C) Association de recherches et publications en management. Tous droits réservés pour tous pays.

La reproduction ou représentation de cet article, notamment par photocopie, n'est autorisée que dans les limites des conditions générales d'utilisation du site ou, le cas échéant, des conditions générales de la licence souscrite par votre établissement. Toute autre reproduction ou représentation, en tout ou partie, sous quelque forme et de quelque manière que ce soit, est interdite sauf accord préalable et écrit de l'éditeur, en dehors des cas prévus par la législation en vigueur en France. Il est précisé que son stockage dans une base de données est également interdit. 


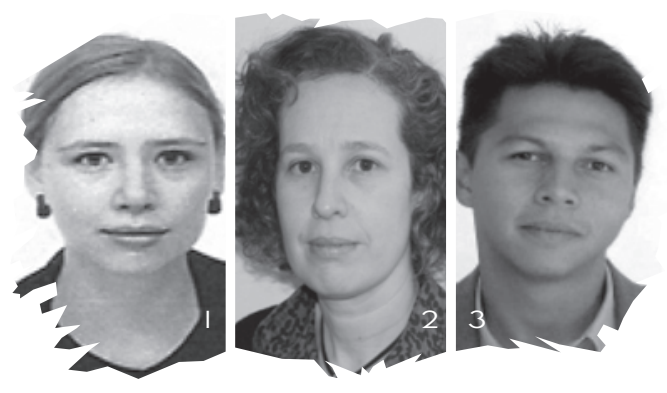

I Elise Bonneveux

ATER

IUT de Tours

2 Isabelle Calme

Maître de Conférences

IAE de Tours

3 Richard Soparnot

Professeur de Management stratégique

Groupe ESCEM

\section{La diffusion d'une démarche RSE innovante au sein d'un réseau de PME : le cas du Centre des Jeunes Dirigeants}

I e développement durable prend de plus en plus d'ampleur face aux évolutions de Lotre société. Il en devient une voie d'entrée et s'apparente à un paradigme nouveau (Pasquero, 2005). En ce sens, il incite l'entreprise à repenser ses modes relationnels et ses pratiques managériales compte tenu des arbitrages souvent difficiles qu'il implique entre l'économique, le social, l'environnemental et le sociétal, entre le court terme et le long terme. Cette perspective d'analyse s'inscrit dans la lignée des développements d'auteurs comme Pasquero (2005), Asselineau et Piré-Lechalard (2008) ou Delpuech (2009), qui invitent à considérer la RSE et son intégration comme une nouvelle manière de gérer l'entreprise; une pratique managériale innovante. Dans cette recherche, il s'agit avant tout de savoir selon quels processus la RSE, comme pratique innovante, peut être diffusée auprès des PME. Car si la grande entreprise (GE) affiche une sensibilité croissante aux pratiques liées au développement durable, il semble plus difficile d'aboutir aux mêmes conclusions pour les PME. Comme le soulignent certains auteurs (Berger-Douce, 2005; Paradas, 2006; Temri \& Fort, 2009), les leviers de diffusion habituellement soulignés dans le cas des GE resteraient majoritairement inefficaces pour favoriser une prise de conscience des dirigeants de PME. Pour ces derniers, il semble nécessaire de trouver d'autres voies. Les principaux travaux sur ces questions pointent certes des voies de diffusion propres à la PME (comme les convictions du dirigeant) mais mettent également en avant d'autres leviers comme les actions collectives menées par des institutionnels tels que les réseaux de pairs ou territoriaux. Ces actions semblent être de plus en plus considérées comme des alternatives prometteuses pour diffuser le développement durable auprès des PME (Quairel et Auberger, 2005; Paradas, 2008). Soulignons également que le discours sur le développement durable émane en majorité de ces acteurs institutionnels. Forts de ce constat, pouvons-nous dire alors que ces espaces collectifs de diffusion constituent des voies privilégiées de diffusion de la RSE auprès des PME ? Et selon quelle(s) logique(s) s'opère alors la diffusion?

Nous nous pencherons donc dans une première partie sur les conditions particulières de la diffusion de la RSE dans les PME. Pour cela nous mobili- 
serons les travaux sur les spécificités de la PME (Julien, 1994; Julien \& Marchesnay, 1996; Torrès, 1999) et sur la diffusion de l'innovation (Callon \& Latour, 1986; Akrich \& al., 1988; Callon, 2006). Dans un second temps, nous exposerons la méthodologie de l'étude, la démarche collective adoptée par le réseau et les logiques de diffusion identifiées. Nous discuterons enfin nos résultats en les confrontant à ceux obtenus lors de recherches menées sur $d^{\prime}$ autres formes collectives de diffusion.

\section{Les conditions de diffusion d'une démarche RSE dans les PME}

Nous opèrerons en deux temps pour appréhender les voies de diffusion de la RSE en PME. Dans un premier temps, nous nous pencherons sur la manière dont les problématiques de RSE s'expriment en PME. Nous montrerons ainsi, que dans le cas des $P M E$, la démarche collective peut offrir un cadre propice à la diffusion de la RSE. Nous mobiliserons dans un second temps les approches sur la diffusion de l'innovation pour mieux cerner le rôle que peuvent jouer ces démarches collectives.
La RSE en PME : le poids des caractéristiques propres à la nature de la firme

Les études menées sur les PME laissent entrevoir des résultats très hétérogènes quant à la réalité des pratiques responsables (Schneider-Maunoury, 2000; Observatoire des PME européennes, 2002; CROCIS-CCIP, 2003; BergerDouce, 2005, 2006, 2008; Quairel \& Auberger, 2005; ACFCl, 2006). Un tel contraste des stratégies responsables des PME - des actions globalement peu intégrées à la stratégie versus des PME militantes qui placent la responsabilité au cœur de leur stratégie - s'explique par le fait que la mise en place d'une démarche particulière de RSE dans I'univers des PME peut dépendre des spécificités de ces organisations (Capron \& Quairel-Lanoizelée, 2004; Lapointe \& Gendron, 2004).

Ainsi, comme l'a précisé Julien (1997): «la PME est une entité propre où les fonctions sont toutes intégrées ou du moins fortement reliées et où le propriétaire-dirigeant en contrôle la plupart des aspects, et pour quelques-unes $y$ participe directement». II est alors possible de résumer les principales caractéristiques de la PME en un mode de fonctionnement intuitif, fortement marqué par le rôle déterminant du propriétaire-dirigeant et par la proximité, que ce soit au sein de l'entreprise ou dans les relations avec les parties pre- 
nantes externes (Julien, 1994; Julien \& Marchesnay, 1996; Torrès, 1999).

Dans ce cadre, l'intégration de la RSE en PME va donc fortement dépendre des représentations que s'en fait le dirigeant. Ainsi la conviction du dirigeant (Paradas, 2006), la recherche de légitimité (Marchesnay, 2009)... peuvent constituer des leviers d'engagement responsable déterminants en PME. Cependant, la forte personnalisation conduit le dirigeant à développer des relations interpersonnelles avec son environnement - la PME fonctionne ainsi en réseau. Qu'il soit social, professionnel ou territorial, le réseau représente ainsi une source de confiance, de sécurité et de flexibilité (Capiez, 2007). II constitue plus particulièrement «un cadre privilégié de réflexion permanent et évolutif pour le dirigeant» (Fourcade, 2008). Comme le précise Julien (1996), «plus les PME sont reliées à des réseaux informationnels riches, plus elles peuvent saisir les opportunités avant les autres tout en s'assurant d'informations essentielles pour compléter leurs ressources effectives ou potentielles». Ainsi, pour Auberger et Quairel (2005), BergerDouce (2006) et Bonneveux et Saulquin (2009), il devient alors un vecteur d'intégration des démarches de RSE en PME. D'ailleurs, au cours des dix dernières années, les démarches collectives dédiées aux enjeux sociétaux se sont multipliées auprès des PME (Berger-Douce, 2006, 2008). Celles-ci prennent généralement la forme de campagnes d'information, de sensibilisation et/ou de formation professionnelle et sont généralement proposées par les chambres consulaires, les agences régionales ou encore par les dirigeants eux-mêmes réunis au sein d'un réseau professionnel.

In fine, la diffusion de la RSE dans les PME repose certes sur les représentations que s'en font les dirigeants mais elle ne peut être appréhendée sans tenir compte de leur encastrement dans des réseaux d'acteurs formant leur environnement direct (Granovetter, 1985; Johannisson \& al., 1994). Dans ces conditions, I'analyse de la diffusion de la RSE en PME doit dépasser le cadre strict de la PME pour s'appuyer sur des approches prenant mieux en compte le rôle des réseaux. II nous semble alors intéressant de nous tourner vers une approche sociologique de la diffusion des innovations.

\section{La RSE comme innovation}

Parce que la RSE constitue une nouvelle manière d'appréhender l'entreprise et conduit à repenser ses modes relationnels et ses pratiques managériales compte tenu des arbitrages entre l'économique, le social, l'environnemental et le sociétal, ellepeut s'analyser sous l'angle de l'innovation. Ce faisant, la démarche RSE peut entraîner des changements pour l'entreprise (nouveaux procédés, produits et services et 
donc nouvelles connaissances et compétences) et/ou pour l'environnement de celle-ci (nouvelles règles du jeu et nouveaux modèles d'affaires). Si l'on pose que la RSE s'apparente à une innovation, comprendre comment elle se diffuse au sein d'un réseau de PME suggère de mobiliser les travaux de Callon et Latour (1986), Akrich et al. (1988) et Callon (2006).

Selon ces auteurs, le succès d'une innovation et donc sa diffusion n'a que peu à voir avec ses caractéristiques intrinsèques. En réalité, seul le réseau socio-économique est au cœur des mécanismes de diffusion d'une innovation. Ainsi, celle-ci se diffuse d'autant mieux qu'elle bénéficie du soutien que lui apportent les acteurs concernés (par exemple les fabricants de produits liés, les fournisseurs, les distributeurs, les prescripteurs, les acheteurs potentiels, les experts, les communautés scientifiques, les organismes de financement...). C'est donc par la constitution d'alliés que l'innovation parvient à s'imposer sur le marché car ceux-ci s'en font les promoteurs. Comment alors se constitue un réseau d'alliés?

Pour Callon et Latour (1986), Akrich et al. (1988) et Callon (2006), la constitution et le soutien d'un réseau d'alliés proviennent des mécanismes d'intéressement. Il s'agit de tisser des liens avec les acteurs susceptibles d'être concernés par l'innovation. Ces derniers, en fonction de leurs intérêts respectifs, vont infléchir l'innovation en cours et générer des adaptations. L'innovation va donc prendre sa forme définitive au fil des interactions sociales entre acteurs. Dès lors, l'innovation pourra leur servir dans le cadre de projets et d'opportunités qui leur sont propres. Eł pour cette raison, ils lui fourniront le soutien nécessaire à sa diffusion. En d'autres termes, c'est parce que les membres du réseau ont été impliqués très en amont du processus de conception de l'innovation qu'ils la soutiennent. D'une certaine manière, ils en deviennent les sponsors, ce qui accélère la diffusion sur le marché. Ainsi émerge un modèle non linéaire de la diffusion de l'innovation : le modèle «tourbillonnaire».

Dans cette optique, la RSE ne doit pas être appréhendée comme une innovation prédéfinie et systématiquement vouée au succès. Elle va fondamentalement prendre forme au fur et à mesure des interactions entre le dirigeant de PME et les membres du/des réseau( $\mathrm{x}$ ) au(x)quel(s) il appartient. Mais elle ne se diffusera qu'à la condition qu'elle dispose d'alliés; les porte-parole et les relais de la RSE dans le réseau.

Ainsi, compte tenu de la forte personnalisation de la PME et des relations de proximité qu'elle entretient avec son environnement, les actions collectives visant à promouvoir des démarches responsables offrent un cadre particulièrement propice à l'étude de la diffusion de la RSE en PME. II s'agit à 
présent de mieux cerner comment cette diffusion s'opère effectivement. Nous allons pour cela présenter l'étude que nous avons menée au sein d'un réseau de PME : le Centre des Jeunes Dirigeants (CJD).

\section{La méthodologie et le cadre de l'étude}

Dans un premier temps, nous exposerons la méthodologie adoptée et l'étude réalisée puis nous présenterons le CJD et le parcours de performance globale?.

\section{Présentation de l'étude}

L'objet de notre étude consistant à comprendre sous quelles conditions une action collective peut avoir un effet d'entrainement auprès de dirigeants de PME, nous avons observé le parcours de plusieurs entreprises, membres du CJD. Dès lors, notre travail de recherche s'inscrit dans une démarche qualitative à visée exploratoire (Charreire \& Durieux, 2003). Nous avons mené des entretiens auprès des différents adhérents et membres du bureau du CJD de Tours (en France). Parallèlement, nous avons rencontré régulièrement 4 entreprises du réseau (PME 1, 2, 3 et 5). II s'agit des entreprises qui ont participé au concours de la Performance Globale $\odot$ entre 2006 et 2010. Nous avons ainsi élaboré une grille d'entretien structurée autour des thèmes suivants :

\begin{tabular}{|l|}
\hline \multicolumn{1}{|c|}{ Thèmes évoqués durant les entretiens } \\
\hline Présentation de l'entreprise \\
\hline Raisons de l'engagement au CJD \\
\hline Perception de la RSE avant l'entrée au CJD \\
\hline Implication actuelle dans le réseau \\
\hline Implication envisagée dans le réseau \\
\hline
\end{tabular}

La collecte des données s'est effectuée par entretiens individuels semi-directifs. Chaque entretien a duré en moyenne une heure et demie. Concernant les 4 entreprises suivies durant leur participation au concours, nous avons mené en moyenne 3 entretiens de deux heures chacun entre fin 2008 et mai 2010. Le tableau en annexe 1 retrace la fiche signalétique de chaque entreprise interrogée. 
Présentation du CJD et du parcours de performance globale

Le CJD est un mouvement patronal né en 1938 pour défendre des valeurs et des actions prônant un libéralisme responsable au sein de la société. II regroupe aujourd'hui 106 agences locales réparties sur le territoire français ainsi qu'à l'international et plus de 3.600 membres, principalement des dirigeants ou cadres dirigeants qui disposent d'une autonomie en matière de management et de gestion. La Figure ci-dessous présente les principales caractéristiques du CJD.

Figure 1 : Présentation des actions du CID en faveur de la performance globale.

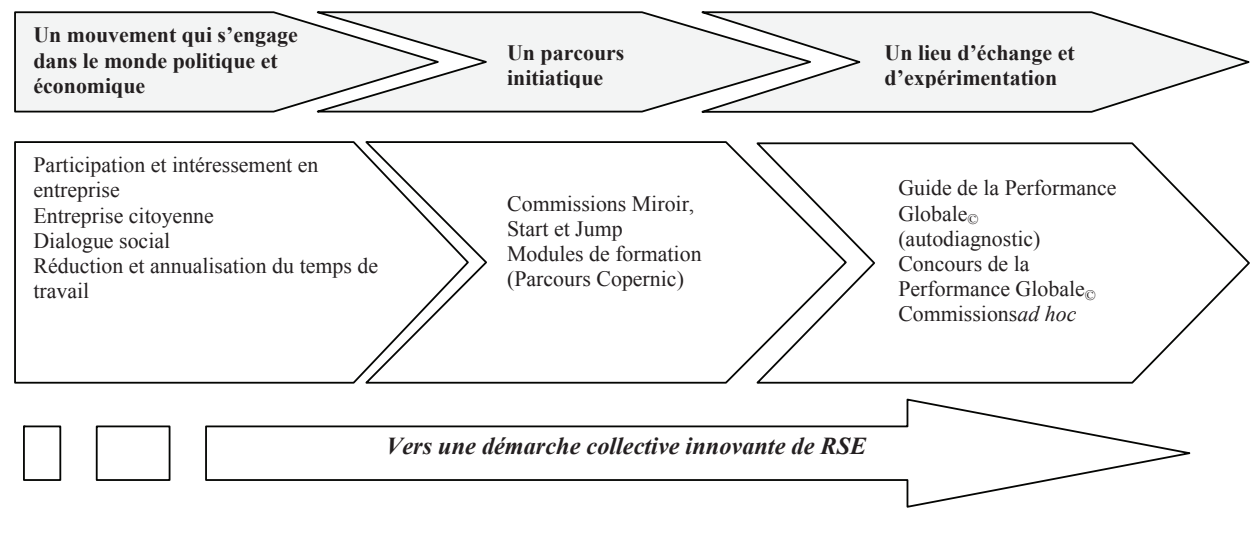

La démarche du réseau se structure autour de réunions plénières, d'actions collectives en lien avec la RSE et d'un parcours intitulé Copernic qui propose un cursus de formation d'une durée de 18 mois afin de donner aux dirigeants les outils nécessaires pour mener une réflexion stratégique sur leur entreprise. Ces actions peuvent être proposées et animées par quelques membres du réseau au gré de leurs besoins afin de mener des réflexions en commun sur un problème similaire rencontré (commis- sions ad hoc). Pour accompagner les entreprises de son réseau, le CJD offre égalementun ensemble d'outils, dont en font partie le guide et le concours de la Performance Globale $_{\odot}$, orientés vers une vision de l'entreprise plus respectueuse des parties prenantes. $\mathrm{Ce}$ dernier propose aux entreprises volontaires de permettre à une équipe d'étudiants issus d'école de commerce ou de master universitaire en management d'établir un diagnostic de la performance globale et de proposer 
des actions concrètes d'amélioration. Pour cela, l'entreprise et les étudiants peuvent s'appuyer sur le guide de la Performance Globale $\odot$.

Le parcours de performance globale du CJD ainsi présenté s'inscrit bien, à notre sens, dans une démarche collective à visée d'innovation dans la mesure où elle incite les adhérents à mener une véritable réflexion stratégique sur leur entreprise. Elle se distingue de celles d'autres réseaux tels les réseaux territoriaux ou les réseaux dits sociaux (association sportives, clubs professionnels...) (Baillette, 2003). Si ces derniers offrent des lieux d'échanges et $d$ 'informations utiles aux dirigeants, leurs actions relèvent davantage d'un service de réseau (Duchéneaut, 1996) et ne portent pas nécessairement sur la formation et la réflexion. La réflexion et les échanges entre homologues impliquent un apprentissage de la confiance entre membres (Chappoz, 1995; Filion 1997). Le réseau de pairs dont fait partie le CJD constitue ainsi un lieu particulièrement propice à ces échanges. II est donc intéressant de s'interroger sur la manière dont s'opère la diffusion de la RSE au sein de ce réseau.

\section{La démarche de RSE au sein du CJD : les con- stats et les logiques déterminants de diffu- sion}

Une lecture interprétative des discours des dirigeants interviewés nous a permis, dans un premier temps, de repérer différents profils responsables au sein des membres du CJD, correspondant à des degrés variés de sensibilisation aux enjeux de la RSE. Dans un second temps, une analyse diachronique de quatre parcours de dirigeants évoluant au sein du réseau nous a conduits à mieux cerner les facteurs explicatifs des différences constatées, mettant ainsi en lumière des logiques dominantes de diffusion des principes RSE au sein du réseau.

\section{Des profils responsables contrastés au sein du CJD}

Nos résultats soulignentdes différences marquantes entre les dirigeants membres du réseau, dans la représentation qu'ils se font de la RSE, dans la manière $d$ 'intégrer ses principes dans leur réflexion stratégique et de les contextualiser dans leur entreprise. Quatre groupes de dirigeants ont été ainsi repérés. 
Les Attentistes (PME 8, 12, 13, 15, 16) : Leur adhésion au CJD est avant tout motivée par le fait de rompre avec le sentiment $d$ 'isolement du chef d'entreprise. Le besoin d'appartenance à un groupe de «pairs» qualifié pour échanger, avoir des réflexions poussées et proposer des solutions concrètes est déterminant. Leurs valeurs sont très proches de celles du CJD, ce qui a généralement facilité leur adhésion au réseau. Néanmoins, ce qui importe pour eux, c'est avant tout la formation proposée au sein du réseau, tournée vers le dirigeant.

PME 12 : "J'ai intégré le CJD à un moment où j'avais des problèmes internes et je cherchais des réponses pour résoudre ces problèmes à court terme. Par contre, je sais que le CJD peut m'apporter beaucoup dans une logique de réflexion sur moimême, de réflexion sur l'entreprise et sur des choix stratégiques. Je pense que le CJD permet autant de résoudre des problèmes liés à son entreprise qu'à se projeter à plus long terme».

Les principes de la performance globale tels que définis par le CJD ne font pas partie de la réflexion stratégique des attentistes. Ces dirigeants sont davantage dans une réflexion à court terme où il est urgent de trouver des solutions à leurs problèmes. Les actions responsables qu'ils mènent restent bien souvent ponctuelles.

On trouve parmi les attentistes principalement des nouveaux adhérents. Ces derniers participent tous à la formation
Valeurs et Performance ainsi qu'à une commission miroir dont l'objectif est de présenter son entreprise aux membres de son groupe - en général un groupe est composé de quatre à cinq dirigeants - et d'exposer une problématique liée au fonctionnement de l'entreprise. La plupart organise également les plénières qui ont lieu au cours de l'année. Certains d'entre eux ont pris part, dès leur entrée dans le réseau, à des actions dédiées à la RSE comme la participation au concours de la Performance Globale $_{\odot}$. II ressort que leur engagement dans une telle démarche s'est fait soit par devoir sociétal soit tout simplement pour avoir un œil extérieur et rechercher la validation des choix stratégiques effectués. Comme en témoignent les discours passés des dirigeants des PME2 et 4 :

PME 2 : "Je venais d'arriver dans l'entreprise et je me suis dit : tiens, ça peut être intéressant pour moi d'avoir un œil extérieur à l'entreprise pour faire en fait mon miroir. Parce que dans une entreprise que I'on crée, on embauche les gens à son image, c'est comme ça. Une entreprise que vous reprenez, c'est les gens qui vous embauchent, ce n'est pas vous, donc il faut s'intégrer dans le groupe et c'est hyper difficile. Le métier de chef d'entreprise, ça ne s'apprend pas. La seule façon de l'apprendre, c'est être dedans, et après, c'est sûr, on peut faire des stages, des cours, c'est ce qu'on fait avec le CJD, et donc c'est pour ça que je voulais intégrer la performance globale. Je me suis engagé dans le concours par devoir sociétal envers le CJD. En tant qu'ancien compagnon, j'ai le souci de transmettre des valeurs et des savoirs aux autres». 
PME 4 : «Je venais d'arriver dans la région et je me suis retrouvé un petit peu embringué là-dedans, démarrant mon entreprise en même temps... et je me suis dit que c'était peut-être une bonne opportunité, justement par rapport à ce que je vous disais tout à l'heure, vérifier qu'on est sur les rails, vérifier que cette vision externe nous renvoie ce qu'on pense avoir mis en œuvre».

Les dirigeants de ces deux entreprises ont depuis évolué sur ces questions de RSE et se retrouvent aujourd'hui dans un autre profil, celui des indécis.

Les Indécis (PME 2, 4, 6, 7, 9, 10, 17) ont pour la plupart participé à la commission Start qui a pour objet d'établir un diagnostic interne et externe de l'entreprise ainsi qu'un business plan sur 5 ans. Ils mènent cette analyse à l'aide du guide la Performance Globale $_{\odot}$ qui comporte des questions sur l'implication de l'entreprise dans les domaines environnementaux, sociaux et sociétaux, ainsi que sur la prise en compte des parties prenantes. Le parcours initiatique ainsi proposé amène les dirigeants à avoir une réflexion plus poussée qui se veut globale, orientée vers une stratégie à long terme de leur entreprise. Elles ont toutes participé au concours de la Performance Globale $_{\odot}$, ce qui leur a permis d'avoir un avis externe sur le fonctionnement de leur entreprise. Pour ce groupe d'entreprises, s'il y a une réelle prise de conscience des principes de RSE et si certaines déjà mettent en place des actions responsables, ces dernières restent souvent limitées à un ou deux volets de la performance globale (le social prédomine). Les actions menées restent bien souvent correctives ou s'inscrivent dans une logique de mise en conformité, sans pour autant amener le dirigeant à repenser profondément ses modes relationnels. Cette prise de conscience ne reste bien souvent qu'au stade de l'éveil.

PME 2 : "Le concours de la Performance Globale $_{\odot} m^{\prime} a-t-i l$ servi? Ce que je sais c'est qu'il m'a ouvert les yeux...il m'a fait comprendre des choses ...que je savais déjà mais en discutant, les étudiants m'ont dit: pourquoi y'a pas ça... ? Et puis on a fini par mettre en place certaines choses, par exemple à l'époque, j'étais tout seul à la direction, ce qui pouvait être dangereux, donc j'ai embauché un adjoint qui me sert de responsable atelier».

PME 4 : «Le concours m'a confirmé que ce que j'avais mis sur le papier dans le cadre de ma stratégie de démarrage n'était pas complètement en dehors de la démarche. Ca c'est un premier point. Deuxième point, elle m'a aussi confirmé que sur des points qui faisaient partie de la démarche que je travaillais, je parle de l'environnemental et du management, soit on avait peu ou pas la main pour nous permettre de répondre aux attentes du concours, soit on était trop petit».

Les Convaincus (PME 3 et 11) sont ceux qui ont, dès le départ, soit une activité en relation directe avec des problématiques de développement durable, soit une conviction assez forte concernant l'implication que leur entreprise doit avoir dans les domaines de l'environnement, du social et du sociétal. De manière générale, ils participent dès la première, voire la deuxième année 
d'adhésion dans le réseau, au concours de la Performance Globale ${ }_{\odot}$ en ayant déjà une ou plusieurs problématiques à soumettre. Ils ont tendance à être plus sensibles aux questions relatives à la performance globale ou à la RSE. Le réseau leur apporte un œil extérieur. II leur permet d'enrichir leurs représentations personnelles de la RSE et les met en quelque sorte en mouvement pour une meilleure prise en compte des enjeux responsables dans le développement de leur entreprise.

PME 3 : "On est convaincu par la démarche...se développer de façon durable, c'est un axe incontournable. J'approuve beaucoup et je trouve ça très intéressant. II y a vraiment quelque chose à faire, en plus pour une PME. Dans les grosses boîtes, c'est intéressant mais ce n'est pas la même chose. Elles vont plutôt voir l'impact. Pour nous, ça a été une sorte de révélateur. Le premier travail a d'abord été sur le social au sein de l'entreprise, et le sociétal avec les partenaires. Et c'est d'essayer d'avancer avec eux. On a mis notamment en place l'intéressement. C'est-à-dire qu'aujourd'hui, on est dans les $2 \%$ des boîtes de notre taille qui donnent du bénéfice aux salariés. Maintenant, on souhaite davantage formaliser nos actions. On est en train de réécrire le business plan pour les trois à cinq prochaines années. Pour nous, c'est la stratégie que l'on place sur le développement durable. D'une vision un peu flove de management qui n'avait pas de nom, c'est passer à quelque chose de concret, structurer autour de l'innovation et de l'exportation»

Les Militants (PME 1, 5 et 14) ont un profil proche des convaincus dans la mesure où, dès le départ, ils sont sensibles aux questions relatives à la
PME 1 : "Au départ je voulais voir ce que c'était la performance globale. Donc j'ai découvert ce que c'était en participant au concours... et mon regard a beaucoup changé depuis que je participe au jury des étudiants, depuis que j'ai organisé cette année la collaboration avec des collègues du CJD, c'est surtout le fait de l'organiser qui m'a aidé à changer.»

RSE et très rapidement participent au concours de Performance Globale ${ }_{\odot}$. La différence porte surtout sur leur engagement dans le réseau et sur les actions menées en son sein. Les militants recherchent davantage à s'impliquer au sein du réseau et plus particulièrement dans des actions orientées vers la RSE. Ils ont en outre tendance à prendre plus rapidement des responsabilités au sein du CJD (mandat de présidence, responsabilités au sein du bureau, animation de commissions, coordination du concours ou encore prise en charge de la communication interne et externe du CJD). L'implication dans le réseau est généralement couplée à une volonté marquée de la part des militants d'améliorer leur entreprise au niveau global. De ce fait, le degré de contextualisation des principes de la RSE dans la stratégie de leur entreprise semble plus élevé, ce qui peut engendrer des changements profonds.

Le dirigeant de la PME 1 a depuis remis à plat sa stratégie en abandonnant l'activité initiale pour développer une autre activité plus respectueuse de l'environnement et plus en lien avec ses convictions. 
PME 14 : «Les plénières, c'est un élément important du partage. J'ai animé une plénière sur l'actionnariat salarial. Je me suis occupé de l'organisation et de l'animation avec mon expert-comptable, j'ai demandé à des salariés de témoigner, c'est un partage. Là, on donne. On reçoit aussi au CJD mais l'idée est aussi de donner un peu de son expérience, de faire profiter de son expérience à d'autres».

PME 5 : "C'est un petit peu ce que le CJD m'a aidé à voir, mon audit de management m'a fait comprendre qu'il faut former les gens, les accompagner si on veut vraiment qu'ils deviennent ce qu'on attend d'eux. Donc, cela a réglé pas mal de choses et ça continue à augmenter le dialogue social. Suite aux préconisations des étudiants durant le concours, j'ai recruté une responsable des ressources humaines, ce qui facilite la communication entre la direction et les salariés».

II ressort de nos résultats des degrés de prise de conscience en faveur de la RSE variés d'un profil à un autre. L'action du CJD semble avoir permis à certains dirigeants d'entrer dans un stade d'éveil en enrichissant leurs représentations de la RSE (cas notamment des indécis). Pour d'autres, l'action du réseau les a «mis en mouvement», prenant conscience de la nécessité de mieux contextualiser les principes de la RSE dans leur entreprise (cas des convaincus). II semble enfin que l'action du réseau ait été un révélateur pour quelques-uns d'entre eux (les militants), prenant conscience que la RSE doit être au cœur de la stratégie de leur entreprise. Tous les adhérents n'ont certes pas la même histoire. En outre ils n'ont pas la même représentation de la RSE. Toutefois, une certaine mise en route vers des préoccupations plus responsables semble se profiler pour une grande majorité d'entre eux, une fois leur arrivée dans le réseau.

Pour tenter de mieux cerner les mécanismes sous-jacents à cet effet d'entraînement, nous avons procédé à une analyse diachronique de quatre parcours de dirigeants au sein du réseau.

\section{Des logiques de diffusions déterminantes repérées}

Grâce au suivi régulier durant trois ans des dirigeants des PME 1, 2, 3 et 5, nous avons retracé leur parcours d'engagement dans la démarche de performance globale proposée par le CJD (cf. encadrés $A, B, C$ et $D$ ). 


\section{ENCADRE A}

De formation "ingénieur ", le dirigeant de la PME 5 prend la succession de l'entreprise familiale en 2003 puis entre au CJD en 2006 dans un but premier d'y rechercher un lieu d'échanges et de rompre le sentiment de solitude propre au métier de dirigeant (profil attentiste). En 2007, il témoigne d'une volonté de contribuer à l'action du réseau en parti-

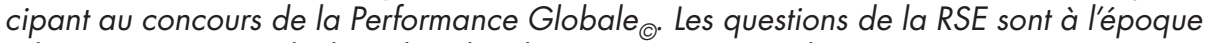
reléguées au second plan. II recherche avant tout un œil extérieur sur son entreprise fortement empreinte d'une culture paternaliste laissée par son père et s'inscrit ainsi dans le groupe des indécis. Les préconisations apportées par les étudiants le poussent alors à établir un audit avec un consultant extérieur. Les contraintes industrielles liées à son activité l'obligent depuis plusieurs années à fonctionner en respectant l'environnement, de ce fait les mesures correctives préconisées tant par les étudiants que par le consultant ont porté en priorité sur le volet social. Une DRH fut embauchée pour prendre en charge la politique salariale de l'entreprise et pour le seconder. Son implication dans le réseau est peu à peu remarquée par ses pairs et le conduit en 2008 à accepter le mandat de président pour deux ans. Au cours de son mandat, le dirigeant mobilise un certain nombre de ressources (....) et décide fin 2009 de participer à nouveau au concours de la Perfor-

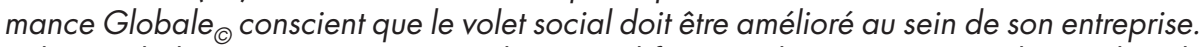
A l'issue de l'année 2010, son mandant prend fin mais il continue à s'impliquer dans le fonctionnement du réseau en participant au côté du dirigeant de la PME 1 à l'animation du concours pour l'année 2011 (rejoignant ainsi le profil militant). L'entreprise poursuit depuis l'intégration du volet social de la RSE dans son entreprise en travaillant en étroite collaboration avec l'ARACT (Association Régionale pour l'Amélioration des Conditions de Travail) sur la mise en place d'une gestion prévisionnelle des emplois et des compétences, sur la recherche de cadences de travail plus adaptées et sur la polyvalence des salariés sur les postes de production. 


\section{ENCADRE B}

De formation gestionnaire, le dirigeant de la PME 1 a d'abord une expérience en tant que salarié pendant plusieurs années dans une entreprise spécialisée dans le redéploiement de sites radio téléphonie. En 2004, ne pouvant évolver dans l'entreprise, il décide de créer sa propre entreprise spécialisée dans la même activité. Se qualifiant d'u écolo convaincu ", c'était un moyen pour lui de fonder une entreprise reflétant ses valeurs. En 2005, il a l'opportunité de rencontrer le CJD et adhère rapidement au réseau, trouvant que les idées et les valeurs étaient en adéquation avec ce qu'il voulait. Très vite il s'implique au sein de la section en proposant d'animer dès 2006 le concours de la Performance Globale(C). Sa mission consiste à coordonner le concours entre les entreprises, le CJD, l'ESCEM et l'IAE de Tours. En 2008, il participe au concours pour avoir un regard extérieur et avancer en cherchant des idées nouvelles pour son entreprise. Suite au concours, le dirigeant a mis en place un certain nombre de mesures correctives (une meilleure formalisation de la stratégie et une recherche d'implication des salariés autour des valeurs, une meilleure transparence dans l'entreprise, il a également engagé des procédures de qualité et de transmission des savoirs). Il a confié à des étudiants en sociologie une enquête sur le climat social de son entreprise. Parallèlement à cela, il a mobilisé des ressources du réseau notamment en participant à des commissions ad hoc et en montant la sienne en 2010. II reconnait aujourd'hui que son implication dans l'animation du concours lui a donné des idées pour faire évolver sa propre entreprise. Suite à des difficultés rencontrées dans son secteur, il a entamé un repositionnement de son activité sur un métier plus sain et plus en lien avec le développement durable et avec ses valeurs. Il est aujourd'hui un fervent militant de la performance globale au sein du réseau et de son entreprise. $80 \%$ de ses salariés viennent au travail en transport en commun ou à pied. Lui-même vient en vélo. Il veille à ce que tout soit éteint la nuit dans l'entreprise, utilise du papier recyclé. L'entreprise a investi dans des toutes petites voitures de façon à ne pas consommer trop d'essence. 


\section{ENCADRE C}

Le dirigeant de la PME 2 reprend une entreprise en 2003 mais se trouve très vite dans une situation difficile puisque quelques mois après la reprise, le chiffre d'affaires diminue de 45\%. Il adhère alors au CJD pour trouver des solutions aux problèmes qu'il rencontre. Il participe en 2006 au concours de la Performance Globale $\odot$ par devoir sociétal mais aussi pour valider des choix passés. De ce fait le diagnostic qui a été posé par les étudiants reflète davantage la propre vision du dirigeant que celle des étudiants. Les mesures correctives ont porté sur quelques réaménagements mineurs dans l'organisation du travail mais les problèmes plus importants liés à la communication interne, à la délégation du pouvoir, à la formalisation de la stratégie ont été étalés dans le temps, soit par faute de moyen, soit par manque de solution à mettre en œuvre. L'embauche d'un adjoint n'a été envisagée que quatre ans plus tard. Dans le même temps, son implication dans le réseau reste réelle mais en retrait par rapport aux dirigeants cités précédemment. En 2010, quatre ans après sa première participation au concours, le dirigeant décide de concourir à nouveau, résolu à ne pas tomber dans les erreurs passées. Il a alors donné carte blanche aux étudiants afin d'avoir un réel miroir de son entreprise, ce qu'il n'avait pas fait la première fois, cherchant à orienter les étudiants sur les points qu'il jugeait importants. Les résultats du second diagnostic montrent que plusieurs points cités déjà dans le premier rapport (manque de communication interne, surcharge de travail et meilleure répartition des responsabilités...) nécessitent encore d'être améliorés. Ce dirigeant reste encore indécis quant aux moyens à mettre en œuvre pour devenir globalement plus performant même s'il reconnait être plus ouvert à ces questions. 


\section{ENCADRE D}

Diplômé en électronique, le dirigeant de la PME 3 crée son entreprise en 2004 dans le domaine de la communication industrielle avec un associé. L'adhésion au CJD leur a été recommandée par un groupement régional qui les avait accompagnés durant quatre ans dans la création et le démarrage de leur affaire. L'entrée au CJD a ainsi été motivée par le maintien d'une certaine continuité : se former et avoir un œil extérieur pour toujours progresser. Ils participent au concours de Performance Globale(C) dès la première année de leur adhésion, principalement par curiosité et pour marquer aussi leur implication dans le réseau (profil proches des attentistes). Le concours devient alors très rapidement un révélateur pour eux. Ils avaient déjà certes pris conscience de l'importance du volet social et sociétal (le volet environnemental dans l'activité qu'ils exercent étant moins déterminant) mais, selon leurs dires, cela découlait même de la nature et de la taille de leur entreprise. Non seulement la démarche proposée par le concours les a renforcés dans cette idée mais elle les a surtout convaincus que la RSE pouvait être intégrée à leur stratégie pour développer l'entreprise par des actions plus responsables. Certaines actions étaient déjà en place dans l'entreprise sur le volet économique et social (mise en place d'une comptabilité par exemple), avec le soutien des étudiants participant au concours, d'autres actions ont alors été développées (réflexion sur les valeurs responsables de l'entreprise et intégration de ces valeurs dans la mise en place d'une vision partagée au sein de l'entreprise, mise en place de l'intéressement, réécriture du Business Plan pour les trois prochaines années). Aujourd'hui cette entreprise fait partie des convaincus du réseau. Etant dans un secteur à forte croissance, la préoccupation première de ce dirigeant est de développer sereinement son entreprise et il ne cherche pas, faute de temps à s'investir davantage dans le réseau, notamment dans le bureau. II reconnaît en outre qu'il aura sûrement besoin de "piqûres de rappel " pour progresser sur ces questions de RSE et n'exclut pas de participer une deuxième fois au concours dans les deux ou trois prochaines années. 
Nous avons visualisé dans la Figure 2 les parcours que nous venons de décrire. Pour cela, nous sommes partis des profils précédemment définis en tenant compte d'un certain nombre de critères comme l'implication des diri- geants dans le réseau (prise de responsabilité), la mobilisation des outils proposés par le CJD (formations, concours de la Performance Globale $\odot_{\odot} .$. ) ou encore le nombre d'années passées dans le réseau.

Figure 2 : Dynamique du processus de diffusion des principes responsables au sein du CJD.

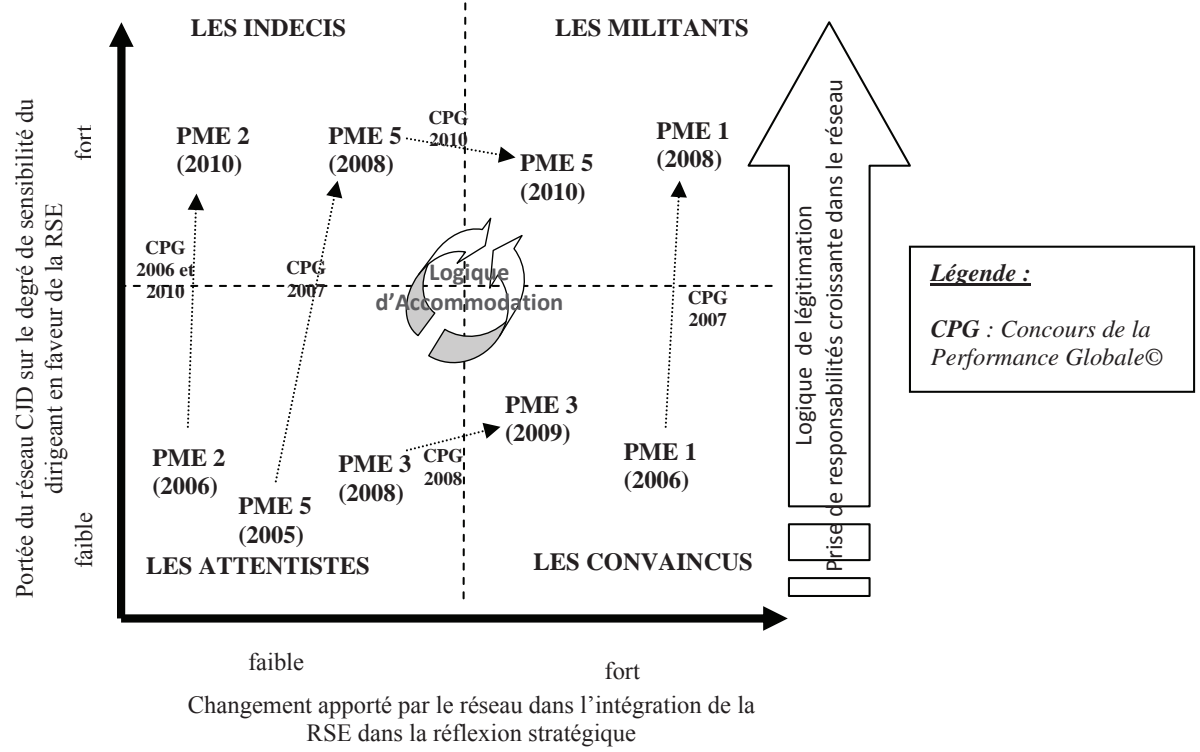

Nos résultats (cf. Figure 2 et encadrés $A, B, C, D)$ montrent que les quatre dirigeants ont tous évolué au sein du réseau vers une prise de conscience plus élevée qu'à leur arrivée, mais à des rythmes et des degrés d'intensité variés. Certains dirigeants ont franchi durant la période étudiée différents stades, correspondant à chaque fois à des profils distincts (cas notamment du dirigeant de la PME 5 : d'attentiste au début, il a fait partie des indécis pour finir militant), tandis que d'autres n'ont franchi qu'un stade pour arriver à des degrés de prise de conscience variés (cas des dirigeants des PME 1, 2 et 3). Nos résultats montrent aussi que le passage progressif d'un stade à un autre n'est pas nécessairement lié au nombre d'année passées dans le réseau. 
En procédant à une analyse comparative des parcours, il ressort que deux mécanismes de diffusion semblent jouer un rôle dans la prise en compte croissante de principes responsables observés chez les quatre dirigeants suivis. Comme le souligne la Figure 2, la diffusion de la démarche de performance globale passe par des mécanismes normatifs (formations professionnelles, autodiagnostic de l'entreprise) et coercitifs (obligation de s'investir dans le fonctionnement du CJD). Ces mécanismes provoquent des comportements mimétiques qui peuvent s'expliquer par la recherche d'une légitimation auprès de l'ensemble des membres du CJD. Ce mimétisme et cette recherche de légitimité touchent plus ou moins rapidement les dirigeants dès leur entrée dans le réseau. Si cette logique de légitimation semble favoriser une prise de conscience pour la RSE, elle n'est pas pour autant une condition suffisante pour que la démarche puisse se diffuser. Elle doit être couplée à une logique d'accommodation qui est rendue possible grâce à des prescriptions réciproques entre acteurs membres du réseau mais également entre membres et acteurs extérieurs (partenaires universitaires et école de commerce). Ces deux logiques semblent se renforcer mutuellement, créant une dynamique d'entraînement au sein du réseau étudié qui conduit les adhérents à une meilleure représentation de la RSE et une meilleure contextualisation de celle-ci dans leur entreprise. Ainsi les différences observées dans les parcours décrits plus haut s'expliquent, à notre sens, par le renforcement mutuel des logiques précédemment citées. En effet, pour la PME 1 et 5, c'est l'activation de différents outils proposés par le réseau couplée à une prise de responsabilité croissante au sein de celuici qui les a entraînés progressivement vers un comportement militant. L'évolution moins marquée de l'engagement responsable des dirigeants des PME 2 et 3 s'explique par le fait que seule la logique d'accommodation semble avoir joué un rôle pour eux. Cette accommodation n'a entrainé des changements que sur une seule dimension : soit sur les représentations du dirigeant (cas de la PME2) soit sur la contextualisation des principes RSE dans l'entreprise (cas du dirigeant de la PME 3).

\section{Conclusion}

Les résultats présentés dans cette recherche montrent que le réseau étudié joue un rôle d'entrainement auprès de ses adhérents en proposant des mécanismes d'intéressement et des dispositifs d'expérimentation qui favorisent la diffusion des démarches responsables proposées.

Ce faisant, cette recherche prolonge les apports de diverses études menées sur la RSE en PME. Nos résultats confirment dans un premier temps I'hétérogénéité 
des comportements responsables des dirigeants de PME (Saulquin \& Schier, 2007; Marchesnay, 2009; Delpuech 2009). Cette diversité de comportements s'explique par les caractéristiques propres aux PME comme les convictions, les représentations des dirigeants (Paradas, 2006, 2007, 2008), la forte proximité fonctionnelle et hiérarchique présente dans ce types de structures (Torrès, 1999, 2003) et l'encastrement territorial (Julien, 1994). Ces caractéristiques constituent bien souvent pour ces auteurs des leviers de diffusion de la RSE déterminants en PME. Au regard de nos résultats, ces leviers ne suffisent pas à eux seuls pour cerner la manière dont les dirigeants s'engagent progressivement dans une démarche de RSE. Le terrain de l'étude, d'ailleurs, aurait pu nous faire supposer des résultats moins contrastés étant donné les fortes valeurs humanistes que véhicule le CJD. Cependant, les dirigeants, indécis ou convaincus, ne mettent pas au premier plan ces valeurs pour justifier leur engagement dans le réseau ou dans une démarche responsable. Les raisons évoquées portent davantage sur la volonté de rompre leur solitude ou de trouver rapidement, grâce aux échanges avec des "pairs», des solutions aux problèmes de fonctionnement de leur entreprise. Ce n'est qu'une fois dans le réseau que ces dirigeants progressent dans leur prise de conscience des enjeux de la RSE, ce qui conduit certains à la considérer comme une nouvelle manière de gérer leur entreprise.
Cela nous a donc amenés, dans un second temps, à rechercher d'autres mécanismes de diffusion, en mobilisant les théories sur la diffusion d'une innovation (cf. les développements dans la partie 1.2). Nos résultats ont ainsi pu mettre en avant, comme l'ont souligné Callon et Latour (1986), Akrichet al. (1988) et Callon (2006), l'importance des mécanismes d'intéressement et la présence d'alliés pour assurer la diffusion de la démarche au sein du réseau. Ces mécanismes d'intéressement prennent la forme, dans le cas étudié, d'une participation aux actions proposées par le réseau (logique d'accommodation), d'une recherche d'implication dans celui-ci, pouvant conduire à une prise de responsabilité dans la gouvernance du réseau en devenant membre du bureau par exemple (logique de légitimation). Notre étude montre en particulier que ces deux logiques favorisent l'apparition d'alliés dans le réseau et ainsi permettent de perpétuer la démarche. A notre connaissance, cette dynamique n'existe pas dans les autres réseaux (groupements territoriaux) qui proposent également des actions collectives de RSE. En effet, ces actions sont souvent ponctuelles et ne concernent qu'un seul volet de la RSE, souvent l'environnemental (Berger-Douce, 2008). Or, dans ce type d'action, le dirigeant n'a pas le temps de s'accommoder aux principes de RSE. L'intégration des principes responsables dans la réflexion du dirigeant et le repositionnement stratégique de l'activité ne s'opèrent alors 
que très rarement. Grâce à la mise en place de mécanismes d'intéressement favorisant une implication et une prise de responsabilité de chacun des adhérents, le CJD offre un espace collectif fédérateur qui semble propice à la diffusion de la RSE dans les PME car bien adapté à leurs particularités et à leur mode de fonctionnement.

Cependant, cette étude invite à une certaine prudence en raison de la nature idiographique du cas étudié. II serait ainsi nécessaire de mener une analyse plus longitudinale dans la mesure où la section du CJD étudiée a depuis triplé son effectif, l'effet d'entraînement s'étant construit autour d'un noyau dur de plusieurs de ses membres. Notre étude mériterait également d'être complétée par des recherches similaires au sein d'autres sections du CJD ou encore auprès d'autres groupements notamment territoriaux afin de valider les mécanismes de diffusion observés dans l'étude. Enfin, des grilles d'analyse complémentaires pourraient être envisagées afin d'acquérir une vision plus complète du phénomène d'intégration des principes de RSE par des dirigeants de PME, membres d'un réseau professionnel, notamment en s'intéressant aux approches appropriatives des outils et dispositifs de gestion responsables. 


\section{Annexe I: Caractéristiques signalétiques des entreprises interrogées}

\begin{tabular}{|c|c|c|c|c|c|c|}
\hline PME & $\begin{array}{l}\text { Secteur } \\
\text { d'activités }\end{array}$ & $\begin{array}{l}\text { Création de } \\
\text { l'entreprise }\end{array}$ & $\begin{array}{l}\text { Contexte dans lequel s'est } \\
\text { effectuée la démarche }\end{array}$ & $\begin{array}{c}\text { Nombre } \\
\text { de salariés }\end{array}$ & $\begin{array}{c}\text { Année } \\
\text { d'intégration } \\
\text { au CJ D }\end{array}$ & $\begin{array}{l}\text { Année de } \\
\text { participation } \\
\text { au concours }\end{array}$ \\
\hline 1 & $\begin{array}{l}\text { Conseil en } \\
\text { performance } \\
\text { énergétique }\end{array}$ & 2005 & $\begin{array}{l}\text { Secteur concurrentiel ; } \\
\text { diversification vers une } \\
\text { activité verte }\end{array}$ & 20 & 2006 & 2007 \\
\hline 2 & $\begin{array}{l}\text { Industrie - } \\
\text { M étallurgie }\end{array}$ & 1947 & $\begin{array}{l}\text { Contexte de reprise difficile } \\
\text { en } 2003\end{array}$ & 20 & 2004 & 2006 et 2010 \\
\hline 3 & $\begin{array}{c}\text { Services et } \\
\text { conseil en } \\
\text { communication }\end{array}$ & 2004 & $\begin{array}{l}\text { Création de l'entreprise en } \\
2003 \text {; accompagnement } \\
\text { par un réseau professionnel }\end{array}$ & 12 & 2008 & 2008 \\
\hline 4 & $\begin{array}{l}\text { Services - } \\
\text { Travail } \\
\text { temporaire }\end{array}$ & 2006 & $\begin{array}{c}\text { Création de l'agence en } \\
2006\end{array}$ & 5 & 1995 & 2007 \\
\hline 5 & $\begin{array}{l}\text { Injection } \\
\text { plastique, } \\
\text { Plasturgie en } \\
\text { réseau }\end{array}$ & 1973 & $\begin{array}{l}\text { Rachat de I'entreprise par le } \\
\text { dirigeant en } 2003\end{array}$ & 95 & 2005 & 2007 et 2010 \\
\hline 6 & $\begin{array}{l}\text { Expertise } \\
\text { comptable }\end{array}$ & 1991 & $\begin{array}{c}\text { A ssocié du cabinet } \\
\text { d'expertise comptable à } \\
\text { Tours }\end{array}$ & 85 & 2005 & 2006 \\
\hline 7 & $\begin{array}{l}\text { Fabrication de } \\
\text { monuments } \\
\text { funéraires }\end{array}$ & 1990 & $\begin{array}{c}\text { Dirigeant de l'entreprise } \\
\text { depuis trois ans, le JD est } \\
\text { sous l'égide d'un } \\
\text { actionnaire unique }\end{array}$ & 15 & 2008 & 2010 \\
\hline 8 & Opticien & 1935 & $\begin{array}{l}\text { Responsable du magasin } \\
\text { depuis deux ans }\end{array}$ & 6 & 2009 & $-\cdots-\cdot$ \\
\hline 9 & $\begin{array}{l}\text { Ingénierie des } \\
\text { systèmes } \\
\text { énergétiques }\end{array}$ & 2004 & $\begin{array}{l}\text { A ouvert l'agence de Tours } \\
\text { en } 2005\end{array}$ & 4 & 2008 & 2009 \\
\hline 10 & $\begin{array}{l}\text { M étiers de } \\
\text { l'installation } \\
\text { électrique }\end{array}$ & 1926 & $\begin{array}{l}\text { L'entreprise compte six } \\
\text { services et } 650 \\
\text { collaborateurs; } \\
\text { le JD est responsable d'un } \\
\text { service de } 52 \text { collaborateurs }\end{array}$ & 52 & 2007 & 2009 \\
\hline 11 & $\begin{array}{l}\text { Fourniture } \\
\text { industrielle et } \\
\text { outillage du } \\
\text { bâtiment }\end{array}$ & 1929 & $\begin{array}{l}\text { Création de l'agence à } \\
\text { Tours en } 1996 \text {; le J D a } \\
\text { repris la gestion de l'agence } \\
\text { en } 2009 \text {; accompagnement } \\
\text { par un consultant pour } \\
\text { analyser les risques } \\
\text { industriels }\end{array}$ & 10 & 2009 & -..-- \\
\hline 12 & $\begin{array}{l}\text { M étiers du } \\
\text { bâtiment, } \\
\text { M açonnerie }\end{array}$ & 1962 & $\begin{array}{c}\text { Le dirigeant a repris } \\
\text { I'entreprise il y a deux ans } \\
\text { et demi }\end{array}$ & 25 & 2009 & ----- \\
\hline 13 & $\begin{array}{l}\text { A ssistanat de } \\
\text { direction }\end{array}$ & 2006 & $\begin{array}{c}\text { Gestion de l'activité en } \\
\text { télétravail ; projets de } \\
\text { développement }\end{array}$ & 0 & 2009 & -.-.-.. \\
\hline 14 & $\begin{array}{c}\text { Conseil et } \\
\text { Ingénierie en } \\
\text { risque industriel } \\
\text { et environnement }\end{array}$ & 2005 & $\begin{array}{l}\text { Dès la création, l'entreprise } \\
\text { a connu une stratégie de } \\
\text { forte croissance }\end{array}$ & 75 & 2008 & 2009 et 2010 \\
\hline 15 & $\begin{array}{l}\text { Conseil en } \\
\text { environnement et } \\
\text { recyclage }\end{array}$ & 2005 & $\begin{array}{c}\text { A ctivité de conseils, de } \\
\text { négoce et de courtage sur le } \\
\text { marché des matières } \\
\text { premières dites } \\
\text { secondaires; clientèle à } \\
\text { l'international }\end{array}$ & 2 & 2009 & -.-.-.- \\
\hline 16 & $\begin{array}{l}\text { Ingénierie et } \\
R \& D \text { en } \\
\text { informatique } \\
\text { industrielle }\end{array}$ & 2007 & $\begin{array}{c}\text { Taux de croissance du } \\
\text { secteur : } \\
\text { plus de } 50 \% \text { par an }\end{array}$ & 2 & 2008 & -...... \\
\hline 17 & $\begin{array}{l}\text { Expertise } \\
\text { comptable et } \\
\text { Conseils }\end{array}$ & 1979 & $\begin{array}{l}\text { Ouverture du cabinet à } \\
\text { Tours en } 2006 \text {; gestion par } \\
\text { trois associés ; croissance } \\
\text { externe en } 2009\end{array}$ & 45 & 2003 & -..-..- \\
\hline
\end{tabular}




\section{Bibliographie}

ACFCl, 2006, «La prise en compte du développement durable et de la responsabilité sociétale de l'entreprise (DD/RSE) par les PME/PMI», Octobre.

AKRICH, M., CALLON, M., LATOUR, B., 1988, «A quoi tient le succès des innovations, premier épisode : I'art de l'intéressement, deuxième épisode : I'art de choisir les bons porte-parole», Annales des Mines, série «Gérer et comprendre».

ASSELINEAU, A., PIRÉ-LECHALARD, P., 2008, "Développement durable et entreprise responsable : une voie pour l'innovation de rupture ?» cahier de recherche $n^{\circ} 8$, ESC Clermont.

BAILLETTE, P., 2003, «Le responsable de PME français et québécois membre d'une association de dirigeants : quelle utilité en matière décisionnelle» Revue Internationale $P M E$, vol. $16, \mathrm{n}^{\circ} 1$, pp. 43-73.

BERGER-DOUCE, S., 2005, «Management environnemental et PME : apports et limites d'une démarche collective», Revue internationale $P M E$, vol. $18, n^{\circ} 3-4$, pp. 93-123.

BERGER-DOUCE, S., 2006, «La démarche collective, un outil d'appropriation du management environnemental par les PME ?», Revue Sciences de Gestion, n 54 , pp. 1-18.

BERGER-DOUCE, S., 2008, «Rentabilité et pratiques de RSE en milieu de PME : Premiers résultats d'une étude française», Revue Management et Avenir, n' 15, pp. 9-29.

BONNEVEUX, E., SAULQUIN J-Y., 2009, «L'appropriation de la RSE par les dirigeants de PME. Le réseau comme vecteur d'apprentissage managérial», Revue Management et Avenir, vol. 3, $n^{\circ} 23$, pp. 170-186.

CALLON, M., LATOUR, B., 1986, «Les paradoxes de la modernité. Comment concevoir les innovations ?», Prospective et Santé, vol. 36, pp. 13-25.
CALLON, M., 2006, "Les réseaux sociaux à l'aune de la théorie de l'acteur-réseau», Sociologies Pratiques, $n^{\circ} 13$, pp. 37-44.

CAPIEZ, A., 2007, «Réseau d'entreprises et performance», Revue Internationale PME, vol. 20, $\mathrm{n}^{\circ} 1$, pp. 41-67.

CAPRON, M., QUAIREL-LANOIZELÉE, F., 2004, Mythes et réalités de l'entreprise responsable, La Découverte, $256 \mathrm{p}$.

CHAPPOZ, Y., 1995, "Le rôle des réseaux d'entrepreneurs dans les processus d'apprentissages managériaux», Actes du 2 ème Congrès International Francophone de la PME.

CHARREIRE, S., DURIEUX, F., 2003, «Explorer et tester : deux voies pour la recherche», in THIÉTART, R.-A., Ed., Méthodes de recherche en management, Ed. Dunod, Paris, 2003, pp. 57-81.

CROCIS-CCIP, Baromètres, 2003, «Le développement durable dans les PME/PMI Franciliennes».

DELPUECH, C., 2009, "Quels sont les facteurs favorisant l'intégration de démarches RSE dans les PME», Actes du RIODD, 25-26 juin 2009.

DUCHÉNEAUT, B., 1996, Les dirigeants de $P M E$ : enquête, chiffres, analyses pour mieux les connaître, Maxima, Paris, 509 p.

FOURCADE, C., 2008, "Stratégies de coopération de proximité : des modes d'innovation organisationnelle en PME», Actes du gème Congrès International Francophone de la PME, L'entrepreneur et la PME, vecteurs de changement et d'innovation, Louvain, 29-31 octobre.

FILION, L. J., 1991, Visions et relations : les clefs du succès de l'entrepreneur, Les Editions de l'Entrepreneur, Montréal, 268 p.

GRANOVETTER, M., 1985, «Economic and Social Structure : The Problem of Embeddedness», American Journal of Sociology, Vol. 91, $\mathrm{N}^{\circ} 3$, pp. 481-510. 
JOHANNISSON, B., ALEXANDERSON, O., NOWICKI, K., SENNESETH, K., 1994, "Beyond anarchy and organization : entrepreneurs in contextual networks", Entrepreneurship and Regional Development, Vol. 6, 1994, pp. 329-356.

JULIEN, P.-A., 1994, Les PME : bilan et perspectives, Ed Economica, Paris, $437 \mathrm{p}$.

JULIEN, P.A., 1996, «Entrepreneuriat, développement du territoire et appropriation de I'information», Revue internationale PME, vol. 9, $n^{\circ} 3-4$, pp. 149-17.

JULIEN, P-A., 1997, Les PME : Bilan et perspectives, GREPME, Ed. Economica, 437 p.

JULIEN, P. A., MARCHESNAY, M., 1996, L'entrepreneuriat, Ed. Economica, $112 \mathrm{p}$.

LAPOINTE, A., GENDRON, C., 2004, «Enseigner la RSE : des recettes utilitaristes à une réflexion critique sur l'entreprise comme institution sociale privée», Actes du 7ème Congrès de I'ADERSE, Toulouse, 21-22 octobre.

MARCHESNAY, M., 2009, "Le petit entrepreneur en développement durable - Essai de typologie», Communication au RIODD, Lille, 2526 juin.

OBSERVATOIRE DES PME EUROPÉENNES, 2002, "Chapitre 9: Responsabilité sociale et environnementale».

PARADAS, A., 2006, «Perception du développement durable par des dirigeants de petites entreprises : résultats d'enquêtes», CIFEPME, Fribourg, Suisse, 25, 26, 27 octobre.
PARADAS, A., 2007, «Le dirigeant comme levier de la RSE en TPE : Approche exploratoire basée sur l'utilisation de récits et d'une cartographie cognitive» Revue Internationale PME, vol. 20, $n^{\circ} 3-4$.

PARADAS, A., 2008, «La position des petites entreprises face à la responsabilité sociale», $R O R, \mathrm{n}^{\circ} 1$, pp. 39-52.

PASQUERO, J., 2005, «La RSE comme objet des sciences de gestion», in Turcotte M.F., Salmon A., Responsabilité sociale et environnementale de l'entreprise, Presses de I'Université du Québec, pp. 80-111.

QUAIREL, F., AUBERGER, M.N., 2005, «Les PME seront-elles socialement responsables ?», in DUPUIS, J.L., LE BAS, C., Le Management responsable, Vers un nouveau comportement des entreprises?, Economica, Paris, pp. 63-84.

SAULQUIN,Y., SCHIER, G., 2005, «Typologie depratiques de RSE», colloque AMS/ISIAM/ IAE Nancy 2, GREFIGE-CEREMO, Agadir, avril.

SCHNEIDER-MAUNOURY, G., 2000, «Des petites entreprises très vertes», L'Expansion Management Review, mars, pp. 77-83.

TEMRI, L., FORT, F., 2009, «Partage des bonnes pratiques de développement durable : le cas des PME agroalimentaires du Languedoc-Roussillon», Innovations, vol. 1, n' 29, pp. 103-125.

TORRÈS, O., 1999, Les PME, Ed. Flammarion, Coll. Dominos, Paris, $128 \mathrm{p}$.

TORRES, O., 2003, «Petitesse des entreprises et grossissement des effets de proximité», Revue Française de Gestion, n' 144, mai-juin, pp. 119 138. 